\section{Tentativas de suicídio em um hospital geral no Rio de Janeiro, Brasil}

\author{
Suicide attempts recorded at a general \\ hospital in Rio de Janeiro, Brazil
}

Guilherme L. Werneck 1,2 Maria Helena Hasselmann 3 Luciana Barreto Phebo 4 Denise E. Vieira 5 Vera Lúcia de O. Gomes 5

\section{Introdução}

Previous suicide attempts are an important predictor of both repeated attempts and suicide. This paper presents the profile of patients who had attempted suicide and were admitted to a general hospital in Rio de Janeiro, Brazil. From April 2001 to March 2002, 160 suicide attempts were recorded (68\% women; $26 \%$ adolescents). Ingestion of pesticides and prescription drugs were the two most common methods used. The two methods had been used by similar numbers of women, while two-thirds of men had used pesticides. As for prevalence of factors associated with attempted suicide, $21 \%$ of patients had been in contact with health services within 30 days prior to the event, $28 \%$ mentioned previous suicide attempts, and 23\% reported other cases of suicide or attempts in the family. Despite the limited data in Brazil, suicide attempts appear to be an important health problem, particularly among youth. Surveillance systems for suicide attempts could help expand knowledge on this problem.

Attempted Suicide; Descriptive Epidemiology; Surveillance
Nos países de economia central, o suicídio tem sido reconhecido como uma prioridade em saúde pública, apresentando-se entre as dez principais causas de morte em todas as idades e entre as três primeiras entre 15 e 35 anos. Adicionalmente, sua incidência vem aumentando na população jovem 1,2,3.

No Brasil, embora a mortalidade por suicídio tenha se estabilizado em torno de 4 mortes por 100 mil habitantes, recente publicação mostrou que, entre 1979 e 1998, as taxas de suicídio aumentaram $43 \%$ entre os jovens residentes nas principais capitais do país 4 . No Estado do Rio de Janeiro, após uma década de declínio, as taxas de suicídio sofreram uma inflexão e, desde 1992, começaram a aumentar progressivamente 5 .

Segundo a Organização Mundial da Saúde 6, diversas circunstâncias produtoras de estresse podem aumentar o risco de suicídio. Dentre estes fatores destacam-se o desemprego, a pobreza, a perda de um ente querido, as discussões familiares ou com amigos, a ruptura de uma relação afetiva e problemas legais ou no trabalho. Ainda que esses eventos sejam relativamente freqüentes, poucos são os indivíduos que, sob essas condições, cometem o suicídio. Neste sentido, é importante considerar a existência de outros fatores predisponentes no processo de determinação do suicídio como, por 
exemplo, o uso abusivo de álcool e drogas, a disponibilidade dos meios para efetuar o ato suicida (exemplo: arma de fogo em casa), a violência física e/ou sexual na infância, o isolamento social e os distúrbios psíquicos como a depressão, a esquizofrenia ou os sentimentos de desesperança 6 . Dentre uma gama muito variada de fatores, a história pregressa de tentativa de suicídio tem sido o evento merecedor de maior destaque na literatura; estimativas apontando para chances 20 a 30 vezes mais elevadas de suicídio em indivíduos que referem este tipo de experiência anterior 7,8 .

No intuito de contribuir para a construção de uma agenda de investigação sobre os eventos relacionados ao suicídio, e considerando a escassa disponibilidade de dados sobre tentativas de suicídio em nosso meio, este artigo apresenta o perfil dos casos de tentativas de suicídio detectados durante o primeiro ano de funcionamento de um sistema de monitoramento para esses agravos em um hospital geral localizado no Município do Rio de Janeiro.

\section{Metodologia}

Em 1999, a Secretaria de Estado de Saúde do Rio de Janeiro instituiu, por meio da Resolução 1.354/1999, a Ficha de Notificação Compulsória de Maus-tratos contra Criança e Adolescente. Em seis meses, mais de vinte tentativas de suicídio haviam sido notificadas por meio dessa ficha, ainda que ela não tivesse sido concebida para esse fim. Mais de 50\% destas notificações originavam-se do Hospital Estadual Getúlio Vargas, um dos maiores hospitais de emergência da cidade do Rio de Janeiro. A identificação desses eventos desencadeou um processo com vistas à implantação de um sistema de monitoramento das tentativas de suicídio. As iniciativas desenvolvidas incluíram a elaboração de ficha de notificação de tentativas de suicídio e panfletos informativos, realização de reuniões e palestras, e contatos institucionais com serviços de saúde mental da região para estabelecer um sistema de referência e contra-referência para esses agravos.

O conteúdo da ficha de notificação de tentativas de suicídio foi decidido com base na literatura sobre os principais fatores associados a esses eventos. Além de dados sócio-demográficos e sobre o método utilizado e as circunstâncias de ocorrência do evento, constam na ficha as seguintes variáveis: tentativas anteriores, história de tentativas de suicídio entre familiares, uso de álcool e/ou drogas ilícitas, presença de deficiência física e visita a serviço de saúde nos últimos trinta dias. Ao final existe um campo para que o respondente (o próprio paciente ou acompanhante) possa relatar livremente suas considerações sobre as possíveis motivações que o levaram a cometer o ato suicida. A notificação do ato suicida é feita ao serviço social por ramal telefônico interno, sendo os membros desta equipe responsáveis pelo preenchimento da referida ficha. A ficha deve ser preenchida sempre que um caso confirmado ou suspeito de tentativa de suicídio dá entrada no setor de emergência do hospital.

Com base nos dados disponíveis na ficha de notificação de tentativas de suicídio, realizou-se este estudo de casos. O levantamento dos dados ocorreu de forma concorrente à ocorrência dos casos e cobriu o período de abril de 2001 a março de 2002. Os dados foram armazenados, processados e analisados em Epi Info 6.04 (Centers for Disease Control and Prevention, Atlanta, Estados Unidos). Testes de quiquadrado de heterogeneidade foram utilizados para avaliar diferenças na distribuição de fatores de risco putativos entre grupos.

\section{Resultados}

No período analisado foram registrados $160 \mathrm{ca}$ sos de tentativas de suicídio, o que corresponde a uma média de 13,3 casos por mês. O mês de maior ocorrência foi setembro, com vinte casos, enquanto o menor número, apenas sete eventos, foi registrado em março. Os dias da semana com maior número de ocorrências foram a sexta-feira e a terça-feira (ambas com trinta eventos), seguidos do sábado (25 eventos) e domingo (24 eventos). A ocorrência de tentativas de suicídios no período entre as 18 horas de sexta-feira e 6 horas da manhã de segunda-feira não foi significativamente mais alta do que seria esperado se os eventos acontecessem aleatoriamente no tempo $(\mathrm{p}=0,60)$.

Do total de casos, $68 \%$ eram do sexo feminino, $26 \%$ adolescentes, $27 \%$ casados ou vivendo em união e $11 \%$ nasceram fora do Estado do Rio de Janeiro. A Tabela 1 apresenta o perfil dos casos segundo sexo. Quase dois terços das mulheres tinham menos de 25 anos de idade, enquanto este percentual foi de apenas $42 \%$ entre os homens. Não houve diferença significativa entre sexos em termos da situação conjugal ou do local de nascimento, mas o perfil de ocupação foi bastante diferente, predominando as estudantes, do lar e aposentadas entre as mulheres e desempregados e trabalhadores manuais ou no setor informal da economia, entre homens. Os principais métodos utilizados fo- 
ram a ingestão de pesticidas (52\%) e medicamentos (39\%). Mulheres tenderam a utilizar os dois métodos na mesma proporção, enquanto cerca de 2/3 dos homens utilizaram pesticidas. Dentre os 51 casos de tentativas com pesticidas em que o agente estava especificado, quarenta e oito decorreram de intoxicações pelo "chumbinho", produto ilegalmente vendido como raticida, que geralmente contém em sua composição o agrotóxico carbamato. Dentre os 19 casos de tentativas com medicamentos em que o agente estava especificado, 17 envolveram drogas psicoativas.

Em relação à prevalência dos fatores de risco para tentativas de suicídio e suicídio habitualmente relatados na literatura, identificouse que $21 \%$ dos pacientes haviam procurado serviços de saúde nos trinta dias anteriores ao evento, sendo que $62 \%$ destes referiram atendimento em serviços de saúde mental. Observou-se, também, que $28 \%$ referiram tentativas anteriores, $23 \%$ referiam casos de tentativas ou suicídio na família, $15 \%$ uso de álcool, $11 \%$ uso de drogas ilícitas, $27 \%$ uso de medicamentos psicoativos e $3 \%$ apresentavam algum tipo de deficiência física.

A Tabela 2 apresenta a prevalência desses fatores segundo a existência ou não de história prévia individual ou familiar de tentativa de suicídio ou suicídio. Dentre os 149 pacientes com informação disponível, 69 (46\%) tinham história pessoal e/ou familiar de tentativas de suicídio. A procura por atendimento médico no mês anterior à tentativa de suicídio foi cerca de três vezes mais freqüente nesse grupo quando comparado com aqueles que não tinham história pessoal e/ou familiar anterior de tentativas de suicídio. O uso de álcool, de drogas ilícitas e o uso de medicamentos psicoativos também foram significativamente mais freqüentes no grupo que referia história pessoal e/ou familiar de tentativas de suicídio.

Em 61 notificações foram registrados os motivos para as tentativas de suicídio. Cerca de $61 \%$ destes referiram os conflitos intrafamiliares como razão para a tentativa, destacando-se as brigas/discussões com os pais ou entre casal, a separação do casal, o fim de namoro e os conflitos com namorado.

\section{Discussão}

Baseando-se nos dados gerados por um sistema de monitoramento de tentativas de suicídio em um hospital geral localizado no Rio de Janeiro, este artigo relata o perfil de 160 casos identificados no período de um ano em termos
Tabela 1

Distribuição de variáveis sócio-demográficas e dos métodos utilizados em casos de tentativas de suicídio segundo sexo, 2001-2002.

\begin{tabular}{|c|c|c|c|c|c|}
\hline \multirow[t]{2}{*}{ Variáveis* } & \multicolumn{2}{|c|}{ Masculino } & \multicolumn{2}{|c|}{ Feminino } & \multirow[t]{2}{*}{$p$ valor } \\
\hline & $\mathrm{n}$ & $\%$ & $\mathrm{n}$ & $\%$ & \\
\hline \multicolumn{6}{|l|}{ Idade (anos) } \\
\hline$<18$ & 2 & 3,9 & 39 & 36,1 & $p<0,01$ \\
\hline $18-24$ & 20 & 38,5 & 28 & 25,9 & \\
\hline $25-34$ & 13 & 25,0 & 21 & 19,4 & \\
\hline $35-49$ & 11 & 21,2 & 17 & 15,7 & \\
\hline$\geq 50$ & 6 & 11,5 & 3 & 2,8 & \\
\hline \multicolumn{6}{|l|}{ Local de nascimento } \\
\hline Estado do Rio de Janeiro & 45 & 86,5 & 89 & 89,9 & $p=0,54$ \\
\hline Outro Estado & 7 & 13,5 & 10 & 10,1 & \\
\hline \multicolumn{6}{|l|}{ Situação conjugal } \\
\hline Casado/União & 35 & 70,0 & 72 & 71,3 & $p=0,87$ \\
\hline $\begin{array}{l}\text { Solteiro/Separado/ } \\
\text { Divorciado/Viúvo }\end{array}$ & 15 & 30,0 & 29 & 28,7 & \\
\hline \multicolumn{6}{|l|}{ Ocupação } \\
\hline Estudante & 5 & 10,4 & 29 & 33,7 & $p<0,01$ \\
\hline Desempregado & 14 & 29,2 & 8 & 9,3 & \\
\hline Do lar/Aposentado & 6 & 12,5 & 22 & 25,6 & \\
\hline Trabalho manual/Setor informal & 17 & 35,4 & 17 & 19,8 & \\
\hline Técnico/Superior & 6 & 12,5 & 10 & 11,6 & \\
\hline \multicolumn{6}{|l|}{ Método } \\
\hline Medicamento & 14 & 26,9 & 47 & 43,5 & $p=0,09$ \\
\hline Pesticida & 33 & 63,5 & 50 & 46,3 & \\
\hline Outros & 5 & 9,6 & 11 & 10,2 & \\
\hline
\end{tabular}

* O total de observações pode variar devido a ocorrência de dados faltantes.

de suas características sócio-demográficas e de alguns putativos fatores de risco. A principal limitação deste estudo é a ausência de um grupo de comparação que forneça informação sobre a distribuição dos fatores de risco na base populacional que originou esses casos. Ainda assim, mesmo considerando suas limitações inferenciais e a possibilidade de sub-registro, a virtual ausência de dados sobre esse tipo de evento em nosso meio justifica o desenvolvimento de estudos preliminares, por exemplo estudos de casos, que possam contribuir para gerar informações básicas a serem exploradas em futuros e mais robustos delineamentos epidemiológicos.

A informação potencialmente mais importante produzida por este estudo diz respeito à freqüência das tentativas de suicídio em nossa população. A identificação de 160 casos de tentativas de suicídio no período de um ano, em 
Tabela 2

Distribuição de alguns fatores de risco e associações com história individual ou familiar de tentativa de suicídio ou suicídio entre casos de tentativas de suicídio, 2001-2002

\begin{tabular}{|c|c|c|c|c|c|}
\hline \multirow[t]{3}{*}{ Variáveis* } & \multicolumn{5}{|c|}{$\begin{array}{l}\text { História individual ou familiar de } \\
\text { tentativa de suicídio ou suicídio }\end{array}$} \\
\hline & \multicolumn{2}{|c|}{ Sim } & \multicolumn{2}{|c|}{ Não } & \multirow[t]{2}{*}{$p$ valor } \\
\hline & $n$ & $\%$ & $\mathrm{n}$ & $\%$ & \\
\hline \multicolumn{6}{|c|}{$\begin{array}{l}\text { Atendimento em serviços de } \\
\text { saúde nos últimos trinta dias }\end{array}$} \\
\hline Sim & 21 & 33,3 & 9 & 11,5 & $p<0,01$ \\
\hline Não & 42 & 66,7 & 69 & 88,5 & \\
\hline \multicolumn{6}{|c|}{ Uso de álcool } \\
\hline Sim & 18 & 26,5 & 5 & 6,3 & $p<0,01$ \\
\hline Não & 50 & 73,5 & 75 & 93,8 & \\
\hline \multicolumn{6}{|c|}{ Uso de drogas ilícitas } \\
\hline Sim & 13 & 19,1 & 3 & 3,8 & $p<0,01$ \\
\hline Não & 55 & 80,9 & 77 & 96,3 & \\
\hline \multicolumn{6}{|c|}{ Uso de medicamentos psicoativos } \\
\hline Sim & 27 & 39,7 & 12 & 15,4 & $p<0,01$ \\
\hline Não & 41 & 60,3 & 66 & 84,6 & \\
\hline \multicolumn{6}{|c|}{ Presença de deficiência física } \\
\hline Sim & 3 & 4,5 & 1 & 1,3 & $p=0,33$ \\
\hline Não & 64 & 95,5 & 78 & 98,7 & \\
\hline
\end{tabular}

* O total de observações pode variar devido a ocorrência de dados faltantes.

apenas um hospital, responsável por menos de $5 \%$ dos atendimentos de emergência da região metropolitana do Rio de Janeiro, sustenta a conjectura de que o problema seja altamente freqüente. Como conseqüência, as relativamente baixas taxas de mortalidade por suicídio no Rio de Janeiro não devem inibir o desenvolvimento de estudos epidemiológicos sobre o comportamento suicida, na medida em que as tentativas de suicídio não só parecem ser o fator de risco mais importante para o suicídio, como apresentam uma carga própria de morbidade para a sociedade.

Outras informações relevantes apresentadas neste estudo concernem aos meios utilizados para tentar suicídio. Destaca-se, em primeiro lugar, a importância do "chumbinho", responsável por cerca de um terço das ocorrências. O "chumbinho" é um produto comercializado de forma ilegal e propalado como um raticida eficiente. Na realidade, este é um nome genérico atribuído a um conjunto de produtos granulados com aparência acinzentada contendo produtos tóxicos, em geral, mas nem sempre, o agrotóxico carbamato ${ }^{9}$. A diminui- ção da oferta do "chumbinho" por meio de uma maior fiscalização sobre sua comercialização e a apreensão do produto, associada à difusão de informações sobre o seu potencial letal e baixo poder rodenticida, poderia reduzir a magnitude e a gravidade desses eventos. Uma segunda informação importante é a alta participação das drogas psicoativas dentre os medicamentos utilizados para tentativas de suicídio, perfil diferente do encontrado em outros estudos internacionais, em que outras drogas como os analgésicos, em particular o paracetamol, aparecem com participação similar aos antidepressivos e tranqüilizantes 10,11 . Isto sugere que $o$ maior controle sobre a prescrição e aquisição de medicamentos psicoativos poderia contribuir para a mudança do perfil de medicamentos utilizados nas tentativas de suicídio, com repercussões potenciais na sua letalidade.

O predomínio das tentativas no sexo feminino e o perfil etário mais jovem das mulheres estão em consonância com a maior parte dos estudos descritivos sobre tentativas de suicídio 6,12 , $13,14,15$. Ainda que informação populacional seja ausente, chama a atenção a proporção de casos com história prévia individual de tentativa de suicídio (28\%), história familiar de tentativa de suicídio ou suicídio (23\%), uso de medicamentos psicoativos (27\%) ou contato com serviços de saúde nos trinta dias anteriores ao evento $(21 \%)$. Os poucos dados disponíveis no Brasil sobre esses comportamentos, como os do inquérito realizado na área urbana de Campinas 16 , apontam para freqüências populacionais bem mais baixas: história prévia individual de tentativa de suicídio $(\sim 3 \%)$, história familiar de tentativa de suicídio ou suicídio $(\sim 10 \%)$, uso de medicamentos psicoativos $(\sim 13 \%)$, sugerindo que as altas prevalências em nossa amostra podem refletir um excesso de exposição entre aqueles que tentam o suicídio. De qualquer forma, todos esses eventos têm sido apontados com possíveis preditores das tentativas de suicídio e suicídio 6,17,18 e merecem investigação mais detalhada em novos estudos epidemiológicos com grupo de comparação apropriado. Devido ao seu alto poder preditivo para novas tentativas, os serviços de saúde podem utilizar a informação sobre história anterior de tentativas de suicídio como um elemento para detecção de grupos com maior propensão para esses agravos.

O uso de medicamentos psicoativos, o contato com serviços de saúde nos trinta dias anteriores ao evento, o uso de álcool e de drogas ilícitas foram mais freqüentes entre indivíduos com história pessoal ou familiar de tentativas de suicídio. Este resultado pode indicar a po- 
tencialidade do uso desses fatores como marcadores de risco tanto para as tentativas como para o suicídio consumado. Entretanto, apesar do consumo de álcool e de drogas ilícitas serem considerados fatores de risco para tentativas de suicídio em diversos estudos 6,19,20, os dados disponíveis nesta investigação não permitem maiores conjecturas, dado que não foram obtidos com base em instrumentos previamente validados.

Assim como neste trabalho, Deslandes 21, em estudo realizado no Rio de Janeiro, mostrou que os problemas de relacionamento familiar ou de namoro e as dificuldades de ordem financeira e/ou os conflitos conjugais estavam presentes na maioria dos casos de tentativas de suicídio estudados.

Ainda que os dados populacionais disponíveis sejam escassos, as tentativas de suicídio parecem ser um evento importante de morbidade, particularmente entre adolescentes e adultos jovens. Sistemas de vigilância para esse agravo se configuram em estratégias para um melhor conhecimento do problema e podem contribuir para evitar novos casos de tentativas ou suicídio consumado. No entanto, é importante salientar que mesmo para aqueles fatores de risco mais explorados na literatura, como o desemprego, os distúrbios do comportamento e as tentativas prévias, pouco se sabe acerca da interação entre esses eventos e sobre os mecanismos pelos quais operam 22 . Neste sentido, mais estudos sobre o tema são necessários, preferencialmente com amostras não baseadas apenas em indivíduos atendidos em serviços de saúde, que utilizem definições mais padronizadas, e de caráter longitudinal. Estudar tentativas de suicídio é um desafio pois envolve recursos consideráveis, instrumentos válidos e muita sensibilidade, mas o custo social associado à decisão de não examinar o problema pode ser irreparável.

\section{Resumo}

A história pregressa de tentativa de suicídio é um importante preditor do suicídio e de novas tentativas. Este artigo apresenta o perfil dos casos de tentativas de suicídio detectados por meio de um sistema de monitoramento para esses agravos em um hospital geral no Rio de Janeiro, Brasil. Entre abril de 2001 e março de 2002 foram registrados 160 tentativas de suicídio, sendo $68 \%$ entre mulheres e $26 \%$ entre adolescentes. A ingestão de pesticidas e o abuso de medicamentos foram os principais métodos utilizados. Mulheres utilizaram os dois métodos na mesma proporção, enquanto $2 / 3$ dos homens empregaram pesticidas. Em relação à prevalência dos fatores de risco para tentativas de suicídio, identificou-se que $21 \%$ dos pacientes haviam procurado serviços de saúde nos trinta dias anteriores ao evento, $28 \%$ referiram tentativas anteriores, $23 \%$ fizeram referência a casos de tentativas ou suicídio na família. Ainda que os dados populacionais disponíveis sejam escassos, as tentativas de suicídio parecem ser um evento importante de morbidade, particularmente entre adolescentes e adultos jovens. Sistemas de vigilância para esse agravo podem ser úteis para um melhor conhecimento do problema.

Tentativa de Suicídio; Epidemiologia Descritiva; Vigilância

\section{Colaboradores}

G. L. Werneck realizou a revisão bibliográfica e participou da análise dos dados. M. H. Hasselmann colaborou na revisão bibliográfica e participou da análise dos dados. L. B. Phebo coordenou a implantação do estudo. D. E. Vieira e V. L. O. Gomes foram responsáveis pela coleta dos dados. Todos autores participaram da concepção do estudo, da interpretação dos resultados e da elaboração do artigo. 


\section{Referências}

1. Monk M. Epidemiology of suicide. Epidemiol Rev 1987; 9:51-69.

2. Bell CC, Clark, DC. Adolescent suicide. Pediatr Clin North Am 1998; 45:365-80.

3. Crawford MJ, Prince M. Increasing rates of suicide in young men in England during the 1980s: the importance of social context. Soc Sci Med 1999; 49:1419-23.

4. Souza ER, Minayo MCS, Malaquias JV. Suicide among young people in selected Brazilian State capitals. Cad Saúde Pública 2002; 18:673-83.

5. Rodrigues NCP, Werneck GL. Age-period-cohort analysis of suicide rates in Rio de Janeiro, Brazil, 1979-1998. Soc Psychiatry Psychiatr Epidemiol 2005; 40:192-6.

6. World Health Organization. World report on violence and health. Geneva: World Health Organization; 2002.

7. Shaffer D, Gould MS, Fisher P, Trautman P, Moreau D, Kleinman M, et al. Psychiatric diagnosis in child and adolescent suicide. Arch Gen Psychiatry 1996; 53:339-48.

8. Gunnell D, Frankel S. Prevention of suicide: aspiration and evidence. BMJ 1994; 308:1227-33.

9. Corrêa CL, Zambrone FAD, Cazarin KCC. Intoxicação por "chumbinho": um desafio para o diagnóstico clínico e para o tratamento. Rev Bras Toxicol 2004; 17:71-8.

10. Hawton K, Townsend E, Deeks JJ, Appleby L, Gunnell D, Bennewith O, et al. Effects of legislation restricting pack sizes of paracetamol and salicylates on self poisoning in the United Kingdom: before and after study. BMJ 2001; 322:1203-7.

11. Simkim S, Deeks J. Co-proxamol and suicide: a study of national mortality statistics and local non-fatal self poisonings. BMJ 2003; 326:1006-8.

12. Schmidtke A, Bille-Brahe U, DeLeo D, Kerkhof A, Bjerke T, Crepet P, et al. Attempted suicide in Europe: rates, trends and sociodemographic characteristics of suicide attempters during the period 1989-1992. Results of the WHO/EURO multicenter study on parasuicide. Acta Psychiatr Scand 1996; 93:327-38.
13. Zhang J, McKeown R, Hussey JR, Thompson SJ, Woods JR. Gender differences in risk factors for attempted suicide among young adults: findings from the third national health and nutrition examination survey. Ann Epidemiol 2005; 15:167-74.

14. Wilcox HC, Anthony JC. The development of suicide ideation and attempts: an epidemiologic study of first graders followed into young adulthood. Drug Alcohol Depend 2004; 76 Suppl:S5367.

15. Hallfors DD, Waller MW, Ford CA, Halpern CT, Brodish PH, Iritani B. Adolescent depression and suicide risk: association with sex and drug behavior. Am J Prev Med 2004; 27:224-30.

16. Botega NJ, Barros MBA, Oliveira HB, Dalgalarrondo P, Marín-León L. Suicidal behavior in the community: prevalence and factors associated with suicidal ideation. Rev Bras Psiquiatr 2005; 27:45-53.

17. Cerel J, Roberts TA. Suicidal behavior in the family and adolescent risk behavior. J Adolesc Health 2005; 36:352.e8-14.

18. Goodwin RD, Beautrais AL, Fergusson DM. Familial transmission of suicidal ideation and suicide attempts: evidence from a general population sample. Psychiatry Res 2004; 126:159-65.

19. Borges G, Walters EE, Kessler RC. Associations of substance use, abuse, and dependence with subsequent suicidal behavior. Am J Epidemiol 2000; 151:781-9.

20. Thatcher WG, Reininger BM, Drane JW. Using path analysis to examine adolescent suicide attempts, life satisfaction, and health risk behavior. J Sch Health 2002; 72:71-7.

21. Deslandes SF. O atendimento às vítimas de violência na emergência: prevenção numa hora dessas? Ciênc Saúde Coletiva 1999; 4:81-94.

22. Welch SS. A review of the literature on the epidemiology of parasuicide in the general population. Psychiatr Serv 2001; 52:368-75.

Recebido em 21/Jul/2005

Versão final reapresentada em 09/Dez/2005 Aprovado em 16/Fev/2006 